\title{
Modified Simplified Rate Equation Model of Flowing Chemical Oxygen-Iodine Laser and its Application
}

\author{
Hai-Xing Yan*1 ${ }^{1}$ Li-Min Hu Zhi Gao \\ (Institute of Mechanics, Chinese Academy of Sciences, Beijing 100080, China)
}

\begin{abstract}
A modified simplified rate equation (RE) model of flowing chemical oxygen-iodine laser (COIL), which is adapted to both the condition of homogeneous broadening and inhomogeneous broadening being of importance and the condition of inhomogeneous broadening being predominant, is presented for performance analyses of a COIL. By using the Voigt profile function and the gain-equal-loss approximation, a gain expression has been deduced from the rate equations of upper and lower level laser species. This gain expression is adapted to the conditions of very low gas pressure up to quite high pressure and can deal with the condition of lasing frequency being not equal to the central one of spectral profile. The expressions of output power and extraction efficiency in a flowing COIL can be obtained by solving the coupling equations of the deduced gain expression and the energy equation which expresses the complete transformation of the energy stored in singlet delta state oxygen into laser energy. By using these expressions, the RotoCOIL experiment is simulated, and obtained results agree well with experiment data. Effects of various adjustable parameters on the performances of COIL are also presented.
\end{abstract}

Keywords: chemical oxygen-iodine laser (COIL), homogeneous broadening, inhomogeneous broadening, rate equation (RE) model, Voigt profile function, performance analysis.

\section{INTRODUCTION}

Performance analyses for predicting the output power and the extraction efficiency of a chemical oxygen-iodine laser (COIL) are of importance. A low pressure limit model was used to calculate the output power and the extraction efficiency of a COIL under the condition of low pressure, i.e. inhomogeneous broadening being predominant, to simplify the treatment of problems. ${ }^{1,2}$ However, this approximation cannot predict the spectrum profile and cannot be utilized correctly in the cases of both homogeneous broadening and inhomogeneous broadening being of importance and non-zero frequency-shift. Generally, the gas pressure in a laser cavity of flowing COIL is 1-10 torr. ${ }^{3}$ In this range, there are conditions of inhomogeneous broadening being predominant and both homogeneous and inhomogeneous broadening being of importance. Furthermore, the lasing frequency can vary in some cases, thereby non-zero frequency-shift will be produced.

By considering the above-described problems, a modified rate equation (RE) model that is adapted to both the condition of homogeneous broadening and inhomogeneous broadening being of importance and the condition of inhomogeneous broadening being predominant is presented in this paper. By using this model, effects of temperature, pressure, iodine

1 * Correspondence: Email: hxyan(a)imech.ac.cn; Phone: (86) 10 62554123; Fax: (86) 1062561284. 
density and frequency-shift on the output power and the extraction efficiency of a COIL are presented as well.

\section{FUNDAMENTAL EXPRESSIONS IN COIL}

Similar to doing in Refs. 1,2, it is assumed that the primary flow containing excited state oxygen and the secondary flow containing iodine are premixed well before entering the laser cavity and the temperature and pressure are constant everywhere in the cavity. The dissociation of iodine has occurred upstream of the laser cavity and deactivation in the cavity during the lasing process is negligible. It means that

$$
n_{1}+n_{2}=n
$$

where $n_{1}$ and $n_{2}$ are the population of the excited state $I\left({ }^{2} P_{3 / 2}\right)$ and the ground state $I\left({ }^{2} P_{1 / 2}\right)$ (or the upper and lower laser level) of the atomic iodine, respectively, $n$ is the total particle number of the atomic iodine entering the laser cavity. The concentration of $\mathrm{O}_{2}\left({ }^{1} \Sigma\right)$ is assumed to be negligible. Because the flow velocity is quite high and the cavity length is relatively short, the flow transit time in the cavity is much shorter than the characteristic time for quenching of the excited molecular oxygen $\mathrm{O}_{2}\left({ }^{1} \Delta\right)$, thus the effect of quenching is assumed to be negligible. Based on the above assumptions, the primary reaction in the cavity can be simplified as:

$$
O_{2}\left({ }^{1} \Delta\right) \mathrm{K} \ll \underset{k_{r}}{\stackrel{k_{f}}{\leftrightarrow}} O_{2}\left({ }^{3} \sum\right)+I^{*}
$$

where $k_{f}$ and $k_{r}$ are the forward and reverse rate constant, respectively, $k_{f}=2.3 \times 10^{-8} / T$, the temperature-dependent equilibrium constant $k_{e q}=k_{f} / k_{r}=0.75 e^{401.4 / T}, T$ is the flow temperature, and $I^{*}$ and $I$ denote the upper and lower laser level of atomic iodine, respectively.

The gain expression is ${ }^{4,5}$

$$
g\left\lfloor " \sigma \varphi\left(v, v_{0}\right)\left(n_{2}-\alpha n_{1}\right),\right.
$$

where $g$ is the gain, $\alpha$ is a constant related to level degeneracy, for the COIL $\alpha \approx 1 / 2, \sigma$ is the pressure-dependent stimulated emission cross section in $\mathrm{cm}^{2}, v$ and $v_{0}$ are the laser radiation frequency and the central frequency of the line profile, respectively, $\varphi$ is the Voigt profile function ${ }^{4,5}$

$$
\varphi\left(v, v_{0}\right) \mathrm{K}^{\prime \prime} \frac{\eta^{2}}{\sqrt{\pi}} \int_{-\infty}^{+\infty} \frac{e^{-t^{2}}}{\eta^{2}+(\xi-t)^{2}} d t
$$

where $\eta$ is the broadening parameter, $\eta=\sqrt{\ln 2} \Delta v_{N} / \Delta v_{D}, \quad \xi$ is the frequency-shift parameter, $\xi=2 \sqrt{\ln 2}\left(v-v_{0}\right) / \Delta v_{D}$, and $\Delta v_{N}$ and $\Delta v_{D}$ are the whole widths at half height for the line profile of the Lorentz broadening and the Doppler broadening, respectively.

From Eqs. (1)-(3), it can be deduced that

$$
\left.n_{1}\lfloor\mathbb{E} " n-g / \sigma \varphi) /(\mathbb{k} \ll \alpha) \text { and } n_{2} \npreceq \mathbb{E} " \alpha n+g / \sigma \varphi\right) /(1 飞 \ll \alpha) \text {. }
$$


Furthermore, the rate equations denoting the population variations of the upper and lower laser levels are

$$
\left.\begin{array}{l}
u \partial n_{2} / \partial x=r n_{1}-k_{p} n_{2}-\sigma \varphi\left(v, v_{0}\right)\left(n_{2}-\alpha n_{1}\right) I /(h v) \\
u \partial n_{1} / \partial x=-r n_{1}+k_{p} n_{2}+\sigma \varphi\left(v, v_{0}\right)\left(n_{2}-\alpha n_{1}\right) I /(h v)
\end{array}\right\}
$$

where $k_{p}$ and $r$ are the collision deactivation rate and the pumping rate of the upper laser level, respectively, $u$ is the flow velocity in the streamwise direction. $I$ is the optical intensity, $h v$ is the energy of a COIL photon. In addition,

$$
r \mathfrak{t}^{\prime \prime} k_{f} n_{\Delta} \text { and } k_{p}=k_{r} n_{\Sigma}
$$

where $n_{\Delta}$ and $n_{\Sigma}$ are population of $O_{2}\left({ }^{1} \Delta\right)$ and $O_{2}\left({ }^{3} \Sigma\right)$, respectively.

From Eqs. (3), (5) and (6), it can be deduced that

$$
u \frac{\partial g}{\partial x}+\left[\left(r+k_{p}\right)+(1+\alpha) \frac{\sigma \varphi\left(v, v_{0}\right) I}{h v}\right] g=\left(r-\alpha k_{p}\right) \sigma \varphi\left(v, v_{0}\right) n
$$

For the Fabry-Perot resonator, with the assumption that the gain is equal to the loss in the laser cavity and the distributed loss and aperture loss are independent upon the distance $x$ in the streamwise direction (so-called constant gain approximation), ${ }^{4,5}$ it can be deduced that

$$
g=g_{t h}, \quad d g_{t h} / d x=0 .
$$

In the case of laser radiation frequency coinciding with the central frequency of the line profile i.e. the frequency-shift parameter $\xi=0$, it can be deduced from Eqs. (7) and (8) that

$$
g=K \sigma n \eta \sqrt{\pi} \cdot \frac{\operatorname{erfc} \eta \exp \left(\eta^{2}\right)}{1+I / I_{s} \eta \sqrt{\pi} \operatorname{erfc} \eta \exp \left(\eta^{2}\right)}
$$

where $K=\left(k_{f} n_{\Delta}-\alpha k_{r} n_{\Sigma}\right) /\left(k_{f} n_{\Delta} Ł \ll k_{r} n_{\Sigma}\right)$ is a parameter which is introduced for writing convenience, $K \sigma n \eta \sqrt{\pi}=g_{0}$ is the small-signal gain; the saturated optical intensity $I_{s}=2 h v k_{r}\left[O_{2}\right]\left[\left(K_{e}-1\right) Y+1\right] /(3 \sigma)$ (where $Y$ is the yield of the molecular oxygen in the singlet delta state $O_{2}\left({ }^{1} \Delta\right)$ and is defined as a fraction of total oxygen population, $Y=n_{\Delta} /\left[\mathrm{O}_{2}\right],\left[\mathrm{O}_{2} \mathrm{~K}^{\prime \prime} n_{\Delta}+n_{\Sigma}\right.$ is the total population of the molecular oxygen).

When $\eta>>1$, i.e. the homogeneous broadening being predominant (high pressure limit), expanding the error function in Eq. (9) gives approximately

$$
g=K \sigma n /\left(1+I / I_{s}\right) .
$$

When $\eta<<1$, i.e. the inhomogeneous broadening being predominant (low pressure limit), Eq. (9) gives approximately 


$$
g=\eta \sqrt{\pi}(K \sigma n) /\left(1+I / I_{s} \cdot \eta \sqrt{\pi}\right)
$$

\section{CALCULATION OF OUTPUT POWER AND EXTRACTION EFFICIENCY}

The output power $P$ can be expressed as ${ }^{1,2}$

$$
\begin{gathered}
P € " P_{a v} \eta_{e x t}, \\
\eta_{e x t}=\eta_{e x t m} \cdot \eta_{e x t r},
\end{gathered}
$$

where $P_{a v}$ is the maximum available power; $\eta_{e x t r}$ is the extraction efficiency of the resonator, which is dependent upon the type of optical resonator, the mirror absorbability and reflectivity and is approximately independent upon the kind and the flow rate of the gas medium and which is a known parameter; $\eta_{\text {extm }}$ is the medium extraction efficiency for extracting power from the gain medium, which describes the availability of the laser medium; $\eta_{\text {ext }}$ is the extraction efficiency or efficiency of the whole system including the optical resonator and gain medium. For a COIL, ${ }^{1,2}$ it can be deduced that

$$
\begin{gathered}
P_{a v}=h v\left[Y_{0}-Y_{t h}\right] Q N_{A}, \\
\eta_{e x t m}=\frac{Y_{0}-Y_{e}}{Y_{0}-Y_{t h}},
\end{gathered}
$$

where $Y_{0}$ and $Y_{e}$ denote yield at the entrance and the exit of the laser cavity, respectively; $Y_{t h}\left(=1 /\left(2 k_{e q}+1\right)\right)$ is the threshold yield; $Q$ is the molar flow rate of oxygen (mol/sec); $N_{A}$ is the Avogadro number. In the following, the medium extraction efficiency is deduced from the expression of gain.

As noted above, it is assumed that quenching of the excited iodine in the cavity is negligible and the molecular iodine has been dissociated completely before entering the laser cavity, so total energy stored in the singlet delta oxygen can be transformed into the laser energy completely ${ }^{1,2}$

$$
u \frac{d n_{\Delta}}{d x}=-\frac{g I}{h v} .
$$

For a Fabry-Perot resonator and based on the constant-gain approximation, ${ }^{4,5} \mathrm{~g}$ is a constant in the streamwise direction $x$. The actual operation pressure in a COIL is quite low and under this condition the expression of gain is relatively simple.

In the case of inhomogeneous broadening, it is deduced from the Eqs. (11) and (16) by using the boundary condition at the cavity entrance $\left.Y\right|_{x=0}=Y_{0}$ that

$$
Y Y^{\prime \prime}\left[Y_{0}-\frac{q}{p} \cdot \exp (-p x)\right]+\frac{q}{p}
$$

where $p$ and $q$ are two parameters introduced for writing convenience and

$$
p=\frac{2 k_{r}}{3 \eta \sqrt{\pi} \sigma u}\left[\frac{\eta \sqrt{\pi} \sigma n}{2}\left(2 K_{e}+1\right)-\left(K_{e}-1\right) g\right],
$$




$$
q=\frac{2 k_{r}}{3 \eta \sqrt{\pi} \sigma u}\left(\frac{\eta \sqrt{\pi} \sigma n}{2}+g\right) .
$$

When expression of the Doppler stimulated excitation cross section ${ }^{3} \sigma_{D}(=\sigma \eta \sqrt{\pi})$ is introduced into the Eq. (17), the obtained distribution of yield $Y$ is completely the same as that in Ref. 1.

In the cases of both homogeneous broadening and inhomogeneous broadening being of importance and/or the non-zero frequency-shift, two approximation expressions of the homogeneous broadening and the inhomogeneous broadening are not adapted and a set of equations composed by Eqs. (7) and (16) and the entrance boundary condition $\left.Y\right|_{x=0}=Y_{0}$ must be solved numerically to obtain the distribution of yield $Y$ and further the extraction efficiency and output power of the laser device.

\section{RESULT AND DISCUSSION}

In the following, a part of results obtained from present modified RE model are compared with the experimental observations in the RotoCOIL. ${ }^{6,7}$ The expression of the resonator extraction efficiency is taken from Refs. 2,6

$$
\eta_{\text {extr }}\left\llcorner^{\prime \prime}\left(1-R_{\text {out }}-S_{\text {out }}\right) /\left[\left(1-R_{\text {out }}-S_{\text {out }}\right)(1+\delta)+S_{\text {out }}+\left(R_{\text {out }} / R_{\max }\right)^{1 / 2}\left(1-R_{\max }\right)\right]\right.
$$

where $R_{\text {out }}$ (variable) is the reflectivity of the outcoupling mirror, $R_{\max }(=0.995)$ is the reflectivity of the highly reflective mirror, $S_{\text {out }}=0.004$ is the scattering coefficient of the outcoupling mirror, and $\delta=0.13$ is the diffraction loss fraction. The threshold gain is dependent upon $R_{\text {out }}$ and $P_{a v}$ is expressed by the Eq. (14). The flow conditions within the laser cavity are deduced from analysis and calculation of the RotoCOIL experiment: ${ }^{6,7}$ the flow temperature $T$ is $167 \mathrm{~K}$, the entrance pressure $P$ is approximately 4 torr, the small signal gain $g_{0}=0.0068 \mathrm{~cm}^{-1}$, the cavity yield $Y_{0}=0.42$, the molar flow rate of oxygen $Q=1.395 \mathrm{~mol} / \mathrm{sec}$, the dilution ratio is approximately $\mathrm{He}: \mathrm{O}_{2}=4: 1$, the flow ratio of $\mathrm{I}_{2}$ and $\mathrm{O}_{2}$ is $I_{2} / O_{2}=0.0158$, flow velocity $u=84100 \mathrm{~cm} / \mathrm{s}$. ${ }^{8}$ In the simulation computation of the experiment, the broadening parameter $\eta=0.08$ is taken in this paper. The calculation herein is based on the RotoCOIL experiment, that is, chosen parameters and their related variables are determined on the base of experimental data and other parameters are directly taken as the experimental data.

Fig. 1 shows a comparison of the result by using present modified model with the RotoCOIL experimental data and results of Ref. 1. It is shown that the agreement is quite well. It is also shown that the present result agrees well with the theoretical result of Ref. 1. Strictly speaking, the model of Ref. 1 is only adapted to the case of lower pressure. However, the Voigt profile is used in present paper thereby the pressure can be extended to a broader range and the present modified model can be used to deal with the problem of both homogeneous broadening and inhomogeneous broadening being of importance.

Fig. 2 shows a comparison of the extraction efficiency of present model for different broadening parameter $\eta$ with result of Ref. 1, here constant flow rates of the lasing medium and the energy-stored medium are assumed. The result of Ref. 1 corresponds to the case when $\eta=0$. The difference of present model result with that of Ref. 1 is quite small when $\eta$ is small; as $\eta$ gets larger, the difference of the two models becomes larger gradually. As $\eta$ increases, the threshold gain at zero power crossing point (denoted by $g_{t h o}$, there exist laser oscillation and output power when $g_{t h}<g_{t h 0}$ ) decreases. 
Thus, when $\eta \geq 0.1$ (correspondingly, pressure $p \geq 4$ torr), it is necessary to consider effects of the homogeneous broadening and the inhomogeneous broadening simultaneously.

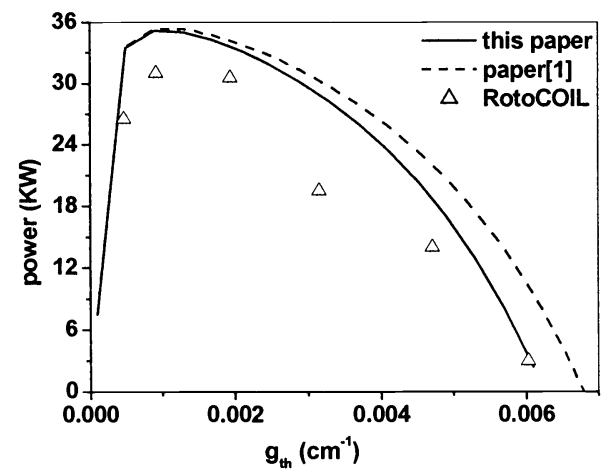

Fig.1. Comparison of theoretical powers with RotoCOIL experimental data.

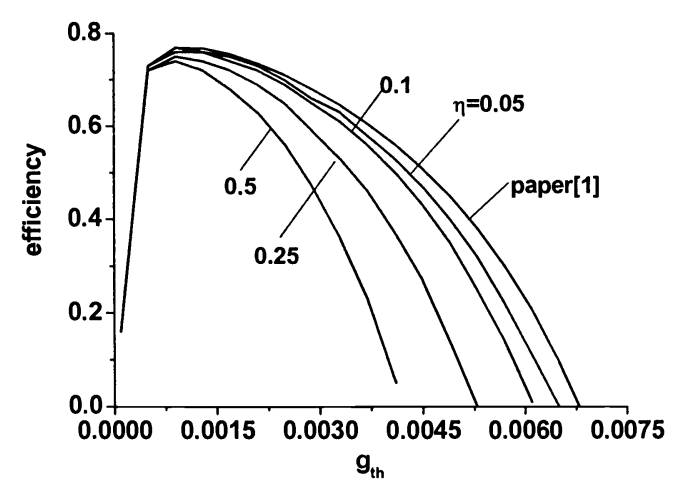

Fig.2. Comparison of efficiencies of Ref. 1 and present paper for different broadening parameter $\eta$.

Fig. 3 shows variations of the output powers with $g_{t h}$ for different flow ratio $I_{2} / O_{2}$ (here the oxygen flux is constant and only the iodine flux varies, the small-signal gain $g_{0}$ varies in proportion to the variation of $I_{2}$ flux). The output power increases with the increment of iodine flux. Therefore, to increase the atomic iodine concentration is favorable for increasing of the output power.

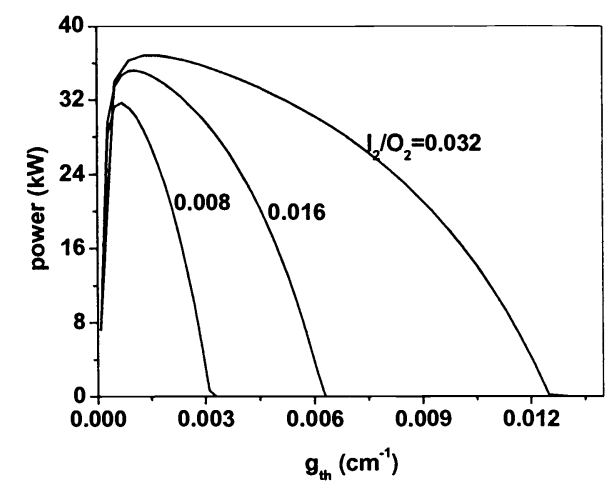

Fig.3. Variation of power with $g_{t h}$ for different $\mathrm{I}_{2} / \mathrm{O}_{2}$ values.

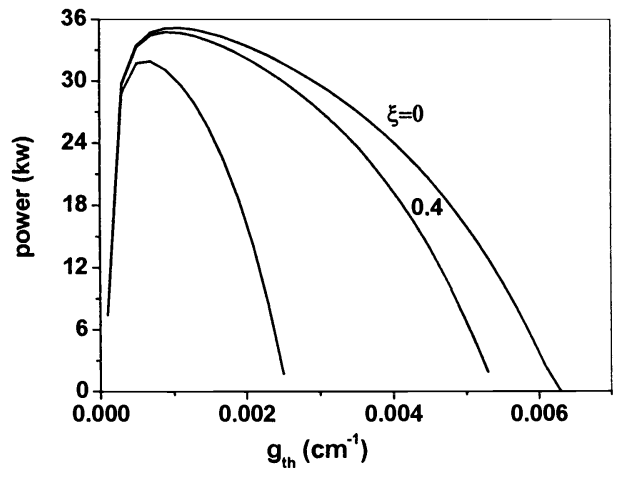

Fig.4. Variation of power with $g_{t h}$ for different frequency-shift parameter $\xi$.

As noted above, the lasing frequency does not always coincide with the central frequency of the spectroscopic line profile and it is possible that a certain frequency-shift exists. Fig. 4 shows variations of the output power with $g_{t h}$ for different frequency-shift parameter $\xi$. It is shown that the output power decreases as the frequency-shift increases. When the frequency-shift parameter $\xi$ reaches 1 , the output power decreases rapidly as the threshold gain increases. 


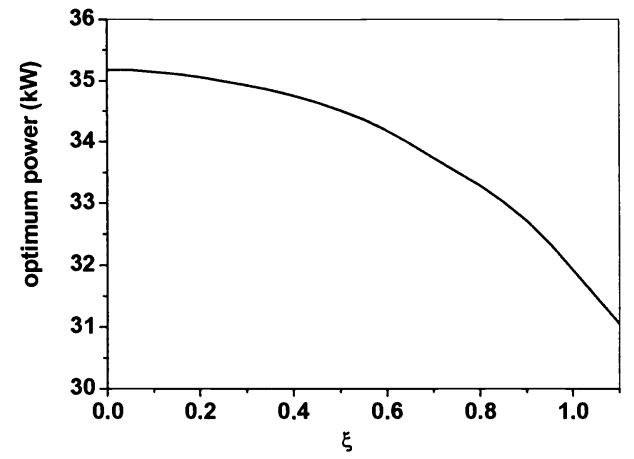

(a)

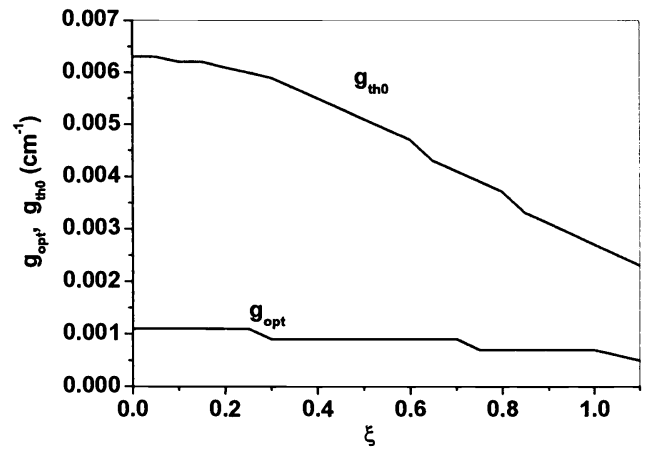

(b)

Fig.5. Variations of the optimum power (a), $g_{\text {opt }}$ and $g_{\text {tho }}(\mathrm{b})$ with frequency-shift parameter $\xi$.

Fig. 5 shows variations of the optimum output power (the output power at the optimum threshold gain), the optimum threshold gain $g_{o p t}$ and the threshold gain at zero power crossing point $g_{\text {tho }}$ with the frequency-shift parameter $\xi$. As the frequency-shift increases, the optimum threshold gain $g_{\text {opt }}$ decreases slightly and the threshold gain at zero power crossing point $g_{t h o}$ decreases gradually. When the frequency-shift parameter $\xi$ is $1, g_{\text {tho }}$ is about one half of that when $\xi$ is 0 . When $\xi$ varies from 0 to 1 , the optimum output power decreases more than ten percents.

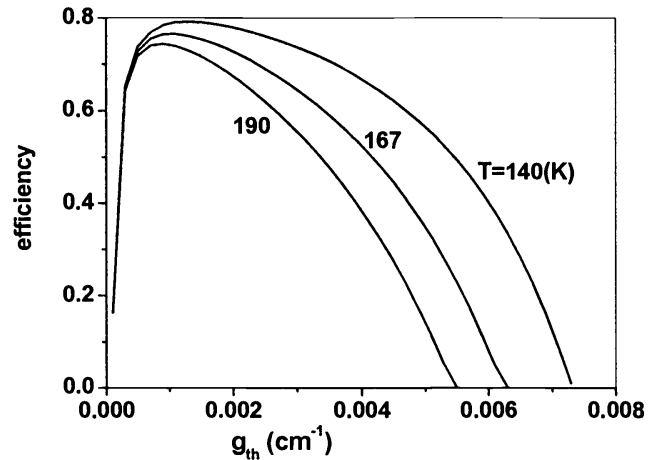

(a)

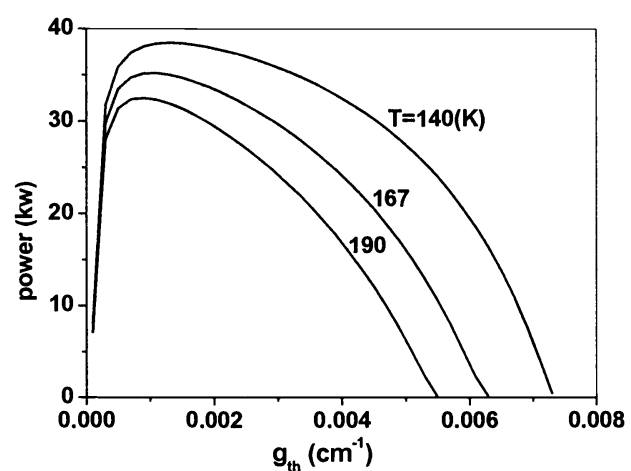

(b)

Fig.6. Variations of power (a) and efficiency (b) with $g_{t h}$ for different temperature $T$.

Fig. 6 shows variations of the output power and the extraction efficiency with the threshold gain $g_{t h}$ for different temperature $T$. Since a decrease of temperature is favorable to excite the atomic iodine from the lower energy level to the excited state, the small-signal gain increases. Furthermore, a decrease of temperature results in an increase of the maximum available power $P_{a v}$. Under the condition of a constant flow rate of molecular oxygen in the excited state, the extraction efficiency increases with a decrease of temperature. Therefore, the lower the temperature is, the higher the output power and extraction efficiency are. In addition, it is also shown that as temperature decreases, the threshold gain at zero power crossing point $g_{\text {tho }}$ increases and the range of threshold gain having higher output power is enlarged, too. Thereby a decrease of temperature by means of increasing the expansion Mach number is an important provision for improving the laser device performances. 


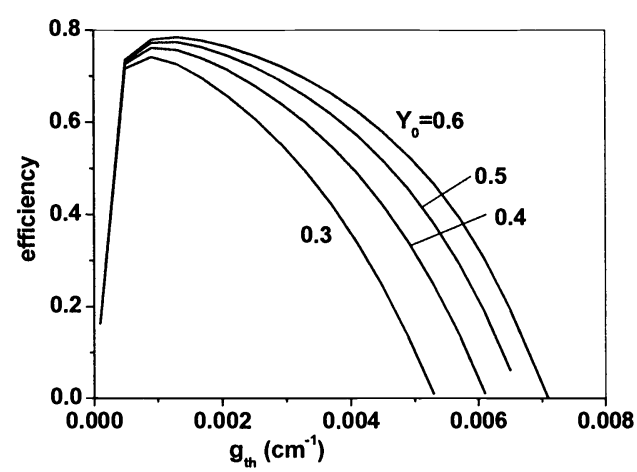

(a)

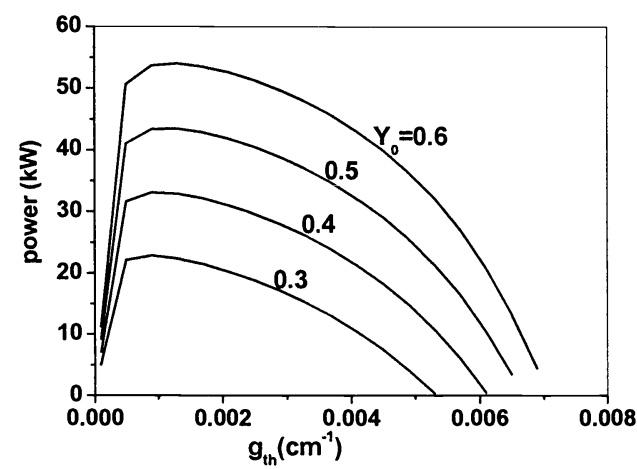

(b)

Fig.7. Variations of efficiency (a) and power (b) with $g_{t h}$ for different $\left.O_{2}{ }^{l} \Delta\right)$ yield at the cavity entrance $Y_{0}$.

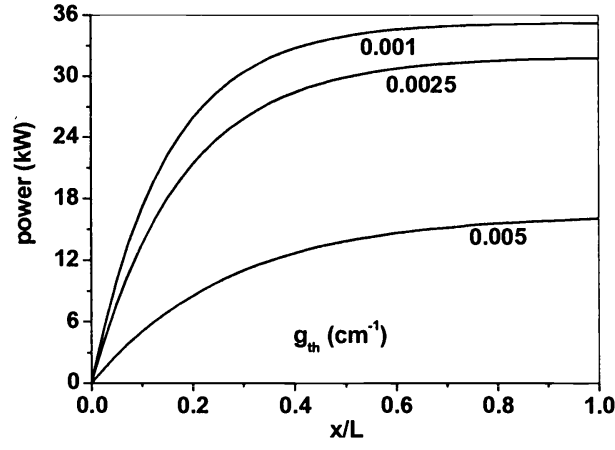

(a)

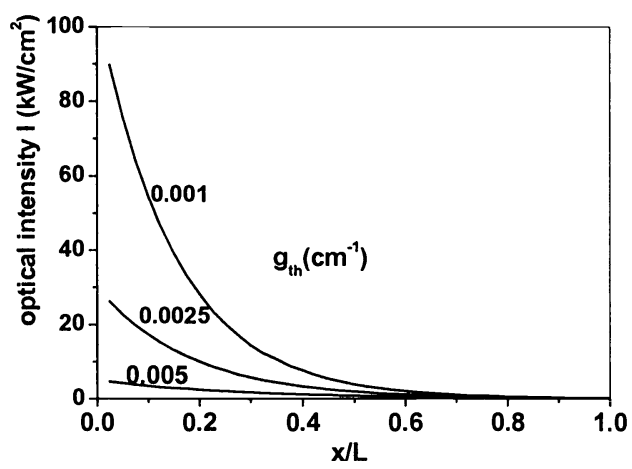

(c)

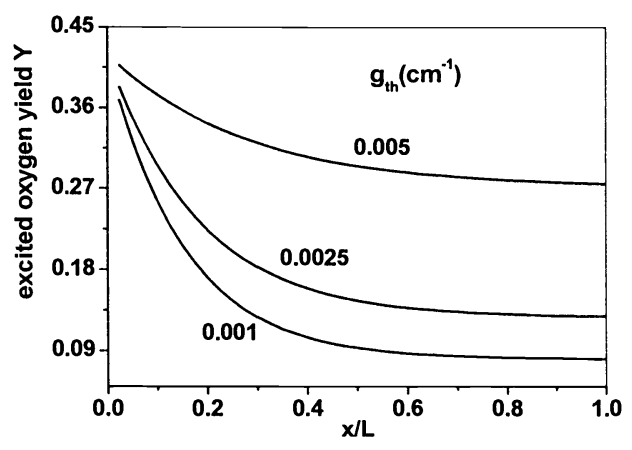

(b)

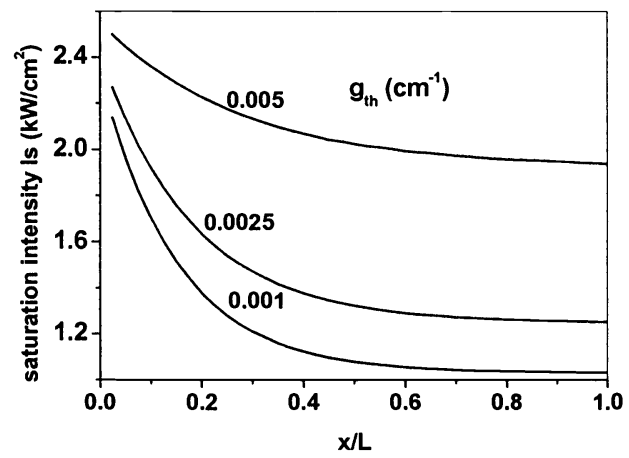

(d)

Fig. 8 Variation of output power (a), $O_{2}(\Delta)$ yield (b), optical intensity $I(\mathrm{c})$ and saturation optical intensity $I_{s}(\mathrm{~d})$ with distance downstream for different $g_{t}$.

Fig. 7 shows variations of the extraction efficiency and the output power with $g_{t h}$ for different yield values of the excited molecular oxygen $Y_{0}$. It is shown that as the yield of $O_{2}(\Delta)$ increases, the threshold gain at zero power crossing point $g_{t h 0}$ increases slightly, the ranges of threshold gain existing output power and having a higher output power are enlarged. The extraction efficiency and output power corresponding to the yield of $O_{2}\left({ }^{l} \Delta\right)$ increase apparently, and the output power increases more obviously. For example, when the yield of $O_{2}(\Delta)$ is increased from 0.3 to 0.6 , the threshold gain at 
zero power crossing point $g_{\text {tho }}$ is increased from $0.0059 \mathrm{~cm}^{-1}$ to $0.007 \mathrm{~cm}^{-1}$ and the extraction efficiency is increased from 0.74 to 0.78 while the maximum output power is increased from $23 \mathrm{~kW}$ to $54 \mathrm{~kW}$, that is, almost $150 \%$. Thus, an increase of the yield of $O_{2}\left({ }^{l} \Delta\right)$ can make a great contribution to increasing the output power.

Fig. 8 shows variations of the output power, the $O_{2}\left({ }^{l} \Delta\right)$ yield, the intracavity optical intensity $I$ and the saturation optical intensity $I_{s}$ with the distance downstream for different threshold gain $g_{t h}$, respectively. Fig. 8(a) shows that as the distance increases, the increase of output power becomes slowly. The output powers increase rapidly at the entrance but almost don't increase near the exit of the cavity. This conclusion is true for all threshold gains, but the higher the threshold gain is, the less the output power is. Fig. 8(b) shows that near the entrance of cavity, the oxygen yields decrease much more rapidly than that near the exit of the cavity. Furthermore, when the threshold gain is higher, more excited oxygen energy stored in the gas flow medium will discharge out of the laser device as waste gas. Fig 8(c) shows that the optical intensity decreases with the distance downstream and the lower the threshold gain is, the higher the optical intensity at the entrance is and the more rapidly the optical intensity with the distance downstream decreases. The optical intensity is decreased to a very small value near the exit of cavity, thus, the optical energy within the gas medium is difficult to be extracted. In Fig. 8(d), the variation of the saturation optical intensity $I_{s}$ with the distance downstream is similar to that of the $O_{2}\left({ }^{l} \Delta\right)$ yield. The lower the threshold gain is, the lower the saturation optical intensity is, i.e. it is easier to reach saturation. As the distance downstream increases, the saturation optical intensity approaches a certain value gradually and the lower the threshold gain is, the lower the saturation optical intensity near the exit of the laser cavity seems to be.

\section{CONCLUSION}

A modified rate equation $(\mathrm{RE})$ model is presented for performance analyses of a flowing chemical oxygen-iodine laser (COIL). This model is utilized to a larger pressure range wherein both the condition of homogeneous broadening and inhomogeneous broadening being of importance and the condition of inhomogeneous broadening being predominant exist. The results agree well with experimental data and previous results. By simulating an experiment with a premixed simplified flow model, present modified model considered the effects of pressure and frequency-shift on the power and extraction efficiency of a COIL that haven't been done in the COIL before. From the calculation results, we can conclude that the increase of pressure, temperature and frequency-shift will reduce the output power and extraction efficiency of COIL, while an increase of the atomic iodine density can increase the output power and the extraction efficiency of the COIL. Distributions of optical intensity and oxygen yield along the flow direction are presented and discussed.

\section{ACKNOWLEDGEMENT}

This research is supported by the National Foundation of Natural Science of China (Project No. 10032050).

\section{REFERENCES}

1. G. D. Hager, C. A. Helms, K. A. Truesdell, D. Plummer, J. Erkkila, and P. Crowell, "A simplified analytic model for gain saturation and power extraction in the flowing chemical oxygen-iodine laser," IEEE Journal of Quantum Electronics 32(9), 1525-1536 (1996).

2. B. D. Barmashenko, and S. Rosenwaks, "Analysis of the optical extraction efficiency in gas-flow lasers with different types of resonator," Appl. Opt. 35(36), 7091-7101 (1996)

3. Q. Zhuang, F.T. Sang, and D.Z. Zhou, Short-Wavelength Chemical Lasers, Beijing: Press of National Defense Industry, (1997). (in Chinese) 
4. R. W. F. Gross and J. F. Bott, Handbook of Chemical Lasers, New York: Wiley, (1976).

5. Z. Gao, "Collisional and inhomogeneous broadening effects in gas-flow and chemical lasers - the theoretical models", Acta Physica Sinica 30(12), 1591-1602 (1981). (in Chinese)

6. J. F. Hon, D. N. Plummer, P. G. Crowell, J. Erkkila, G. D. Hager, C. A. Helms, and K. A. Truesdell, "A heuristic method for evaluating COIL performance," presented at the AIAA $25^{\text {th }}$ Plasmadynamics and Laser Conf., Colorado Springs, CO, USA, June 20-23, paper 94-2422 (1994).

7. K. A. Truesdell, C. A. Helms, and G. D. Hager, "COIL development in the USA," presented at the AIAA $25^{\text {th }}$ Plasmadynamics and Laser Conf., Colorado Springs, CO, USA, June 20-23, paper 94-2421 (1994).

8. R. C. Buggeln, S. Shamroth, A. I. Lampson, and P. G. Crowell, "Three-dimensional (3-D) Navier-Stokes analysis of the mixing and power extraction in a supersonic chemical oxygen-iodine laser (COIL) with transverse $\mathrm{I}_{2}$ injection", presented at the AIAA $25^{\text {th }}$ Plasmadynamics and Laser Conf., Colorado Springs, CO, USA, June 20-23, paper 94-2435 (1994)

9. D.A. Coppland, et. al., "Simple model for optical extraction from a flowing oxygen-iodine medium using a Fabry-Perot resonator," Proc. SPIE Optical Resonators 1224, 474-499 (1990). 